\title{
GCU
}

Glasgow Caledonian

University

University for the Common Good

\section{Quantification of the performance of iterative and non-iterative computational methods of locating partial discharges using RF measurement techniques}

El Mountassir, Othmane; Stewart, Brian G.; Reid, Alistair J.; McMeekin, Scott G.

Published in:

Electric Power Systems Research

DOI:

10.1016/j.epsr.2016.10.036

Publication date:

2017

Document Version

Author accepted manuscript

Link to publication in ResearchOnline

Citation for published version (Harvard):

El Mountassir, O, Stewart, BG, Reid, AJ \& McMeekin, SG 2017, 'Quantification of the performance of iterative and non-iterative computational methods of locating partial discharges using RF measurement techniques', Electric Power Systems Research, vol. 143, pp. 110-120. https://doi.org/10.1016/j.epsr.2016.10.036

\section{General rights}

Copyright and moral rights for the publications made accessible in the public portal are retained by the authors and/or other copyright owners and it is a condition of accessing publications that users recognise and abide by the legal requirements associated with these rights.

Take down policy

If you believe that this document breaches copyright please view our takedown policy at https://edshare.gcu.ac.uk/id/eprint/5179 for details of how to contact us. 


\title{
Quantification of the Performance of Iterative and Non-Iterative
}

\section{Computational Methods of Locating Partial Discharges Using RF}

\section{Measurement Techniques}

\author{
Othmane El Mountassir ${ }^{1 *}$, Brian G Stewart ${ }^{2}$, Alistair J Reid ${ }^{3}$ and Scott G McMeekin ${ }^{4}$ \\ 1 Offshore Renewable Energy Catapult, 121 George Street, Glasgow G1 1RD, UK \\ 2 Department of Electronic and Electrical Engineering, University of Strathclyde, 204 George Street, Glasgow G1 1XW, UK \\ 3 School of Engineering, Cardiff University, The Parade, Cardiff CF24 3AA, UK \\ 4 Institute for Sustainable Engineering \& Technology, Glasgow Caledonian University, 70 Cowcaddens Road, Glasgow G4 0BA, \\ UK
}

\begin{abstract}
1 Partial discharge (PD) is an electrical discharge phenomenon that occurs when the

2 insulation material of high voltage equipment is subjected to high electric field stress.

3 Its occurrence can be an indication of incipient failure within power equipment such as

4 power transformers, underground transmission cable or switchgear. Radio frequency

5 measurement methods can be used to detect and locate discharge sources by measuring

6 the propagated electromagnetic wave arising as a result of ionic charge acceleration. An

7 array of at least four receiving antennas may be employed to detect any radiated

8 discharge signals, then the three dimensional position of the discharge source can be

9 calculated using different algorithms. These algorithms fall into two categories; iterative

10 or non-iterative.

11 This paper evaluates, through simulation, the location performance of an iterative method (the standard least squares method) and a non-iterative method (the Bancroft algorithm). Simulations were carried out using (i) a "Y" shaped antenna array and (ii) a square shaped antenna array, each consisting of a four-antennas. The results show that

15 PD location accuracy is influenced by the algorithm's error bound, the number of iterations and the initial values for the iterative algorithms, as well as the antenna

17 arrangement for both the non-iterative and iterative algorithms. Furthermore, this
\end{abstract}


18 research proposes a novel approach for selecting adequate error bounds and number of

19 iterations using results of the non-iterative method, thus solving some of the iterative 20 method dependencies.

21 Keywords: Partial discharges; Iterative algorithms; Non-Iterative algorithms; Radio 22 Frequency; Fault location; Time difference of arrival. 


\section{Introduction}

24 Radio frequency (RF) measurement technique using receiving antennas can be used to 25 detect the radiated energy from PD sources or any other electrical discharge activities, 26 subsequently facilitating the discharge source triangulation. Using a receiving antenna 27 array, which may be arranged in various forms, the time differences of arrival (TDOA) 28 between received signals on each of the respective antennas allows the 3 dimensional 29 position of the electrical discharge source to be deduced by processing of the TDOA 30 values through iterative or non-iterative location algorithms. The location of partial 31 discharges using emitted RF techniques in HV equipment has been widely investigated 32 [1-5]. Research in this area has been carried out on cables [6-9], gas and air insulated 33 switchgears [10-14] and transformers [15-17]. PD location in cables, and to a degree in 34 gas-insulated substation (GIS), is a two-dimensional problem, while internal localisation within power transformers and localisation in three dimensions in wide-area HV substations requires robust computation algorithms [1].

37 There are two types of computational algorithm which can be used to locate partial 38 discharges in three dimensions; (i) iterative methods and (ii) non-iterative methods. In 39 this study, a non-iterative method was selected due to the large success of these methods 40 in Global Positioning System (GPS) applications such as navigation and location 41 systems. The choice of an iterative method was mainly due their efficiency in solving 42 nonlinear problems involving large number of variables.

43 The iterative methods give an approximate solution to nonlinear equations based on a 44 number of iterations and starting with an initial value, which is improved at each 45 iteration by an error bound until a converged solution is found or until a maximum number of iterations is reached. Taylor expansion and Newton-Raphson techniques are common iterative methods that can be used to solve the equations of nonlinear systems. 
48 These methods have been used in different studies to locate PD [1, 18-19]. The study in

49 [18] highlighted that the performance of the Taylor expansion method depends on the 50 accuracy of the initial values and the number of sensors, whereas the study by [1]

51 showed that the Newton-Raphson method successfully locates PD and that the location 52 accuracy depends on the arrangement of antennas. Study [19] also used the Newton53 Raphson method to locate PD and found that in some cases the algorithm did not 54 provide a converged solution. It indicated that a solution called the "grid search 55 method" which consists of using a range of values within a grid as initial values to 56 determine a converged solution helped improve accuracy. Despite the fact that these 57 studies highlighted the success of these iterative methods to locate discharges activities 58 within a reasonable margin of error, a limited number of published studies have 59 attempted to evaluate fully the performance of non-iterative and iterative methods in 60 their ability to locate accurately the position of electrical discharge sources.

61 In order to evaluate the performance of iterative and non-iterative algorithms, the 62 present study investigates through simulation the location performance of a well63 established iterative method; the standard least squares (SLS) method, and a non64 iterative method; the Bancroft algorithm [22]. Two antenna array configurations ( $\mathrm{Y}$ and 65 square shape), both consisting of 4 antenna positions were chosen for the investigations reported herein evaluating the performance of the respective location algorithms. The

67 square and ' $\mathrm{Y}$ ' array configurations are commonly used and were selected since they 68 have been used in previous studies $[1,4]$ to investigate electromagnetic (EM) wave 69 propagation PD sources.

70 The paper is structured as follows: The mathematical formulation of the SLS and

71 Bancroft location algorithms are presented in Section II; Section III presents the 72 methodologies used in the present study; Section IV presents the results of PD location 
73 studies using the SLS and Bancroft algorithms respectively (in each case two different

74 antenna arrangements were investigated). For simplification, the simulated PD location

75 data points refer to any electrical discharge source emitting EM wave radiation; Section

$76 \mathrm{~V}$ compares the characteristics of both the iterative and non-iterative algorithms used;

77 Section VI proposes a new approach to select adequate error bounds and number of 78 iterations using results of the non-iterative methods; Section VII summarises the 79 findings of the study.

802 Formulation of the SLS and Bancroft Algorithms

81 A minimum of four spatially separated antennas may be used to triangulate the location 82 of a PD event in 3 dimensions using RF methods (Figure 1). Knowing the grid 83 coordinates of each antenna in the array then allows the propagation time from the PD 84 source to the respective antennas to be calculated using the basic formula $D=v . t$, 85 Where $D$ is distance, $v$ is propagation velocity and $t$ is propagation time. This technique, 86 commonly referred to as 'triangulation', is described by Equation (1):

$87 \quad\left(x-x_{i}\right)^{2}+\left(y-y_{i}\right)^{2}+\left(z-z_{i}\right)^{2}=\left(v_{e} \cdot t_{i}\right)^{2}$

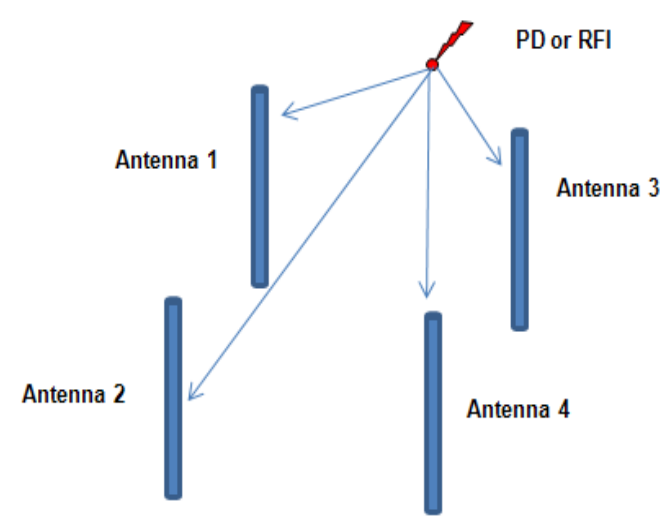

89 Where $\left(x_{i}, y_{i}, z_{i}\right)$ are the coordinates of the $i^{\text {th }}$ antenna in Cartesian space, $(x, y, z)$ 90 represent the true coordinates of the PD event, $\mathrm{v}_{e}$ is the speed of light $\left(3 \times 10^{8} \mathrm{~m} / \mathrm{s}\right)$ and $t_{i}$ 
91 represents the 'time-of-flight' of the propagating PD signal from its source to the $i^{\text {th }}$

92 antenna. It should be noted that since the study is a simulation based investigation, the

93 speed of light was considered to be in a vacuum and that this value changes depending

94 on the insulating material.

95 Let the time-of-flight from the PD source to antenna $A_{1}$ be $T$ and the time-difference-of-

96 arrival between antennas $A_{1}$ and $A_{n}(n=2,3,4)$ be $\tau_{l n}$. Equation (1) now expands into

97 the following four formulae [20]:

$98 \quad\left(x-x_{1}\right)^{2}+\left(y-y_{1}\right)^{2}+\left(z-z_{1}\right)^{2}=\left(v_{e} \cdot T\right)^{2}$

$99 \quad\left(x-x_{2}\right)^{2}+\left(y-y_{2}\right)^{2}+\left(z-z_{2}\right)^{2}=\left(v_{e} \cdot\left(T+\tau_{12}\right)\right)^{2}$

$100\left(x-x_{3}\right)^{2}+\left(y-y_{3}\right)^{2}+\left(z-z_{3}\right)^{2}=\left(v_{e} \cdot\left(T+\tau_{13}\right)\right)^{2}$

$101\left(x-x_{4}\right)^{2}+\left(y-y_{4}\right)^{2}+\left(z-z_{4}\right)^{2}=\left(v_{e} \cdot\left(T+\tau_{14}\right)^{2}\right.$

\subsection{Standard Least Squares (SLS) algorithm}

103 Using on the non-linear equations in (2), the position of a PD source $(x, y, z)$ can be

104 computed using the least squares method given in Equation (3).

$105 S(X)=\sum_{i=1}^{N}\left(Y_{i}(X)\right)^{2}$

106 In least squares, the standard definition of $Y_{i}(X)$ is given in Equation (4). Based on the 107 definition of $\mathrm{Y}_{\mathrm{i}}(\mathrm{X})$, the least squares method minimises the sum of the square of the 108 residuals.

109

$Y_{i}(X)=\sqrt{\left(x-x_{i}\right)^{2}+\left(y-y_{i}\right)^{2}+\left(z-z_{i}\right)^{2}}-\left(v_{e} *\left(T+\tau_{l i}\right)\right)$

110 Since the aim is to compute the values of $x, y$ and $z$ which minimise $S(X)$, the partial

111 derivative of $S(X)$ with respect to $x, y$ and $z$ is calculated with the equation set equal to $O$

112 as shown in Equation (5):

$113 \quad \frac{\partial S}{\partial x}=0, \frac{\partial S}{\partial y}=0, \frac{\partial S}{\partial z}=0$ and $\frac{\partial S}{\partial T}=0$. 
114 Substituting $p$ to represent $x, y$ or $z$, the iterative solution for each coordinate and for $T$

115 becomes:

$116 \quad p=\frac{1}{N} \sum_{i=1}^{N} p_{i}+\frac{1}{N} \sum_{i=1}^{N} \frac{\left(p-p_{i}\right)\left(T+\tau_{1 i}\right) v_{e}}{\sqrt{\left(x-x_{i}\right)^{2}+\left(y-y_{i}\right)^{2}+\left(z-z_{i}\right)^{2}}}$

$117 T=\frac{\sum_{i=l}^{N} \sqrt{\left(x-x_{i}\right)^{2}+\left(y-y_{i}\right)^{2}+\left(z-z_{i}\right)^{2}}}{\sum_{i=l}^{N} v_{e}}-\frac{1}{N} \sum_{i=l}^{N} \tau_{1 i}$

118 Where $N$ is the number of antennae and $\tau_{1 i}$ is the TDOA between a signal measured by

119 the $i^{\text {th }}$ antenna and by antenna 1. For chosen initial conditions, the formulae derived 120 above may be applied iteratively until solutions for $x, y$ and $z$ are converged upon, given

121 a defined error bound and an upper limit on the number of iterations [4, 21].

$122 \quad 2.2$ Bancroft algorithm

123 Developed by Bancroft [22], this algorithm was derived for application to global 124 positioning system (GPS) location. Bancroft's algorithm makes use of the Lorenz inner 125 product for time-space vectors, which is defined considering $u$ and $w$ vectors of the 126 form:

$127 \quad u=\left[\begin{array}{c}x_{u} \\ y_{u} \\ z_{u} \\ v * t_{u}\end{array}\right], \quad w=\left[\begin{array}{c}x_{w} \\ y_{w} \\ z_{w} \\ v * t_{w}\end{array}\right]$

128 Where $x, y$ and $z$ are the coordinates of the two vectors $u$ and $w, v$ is a constant which 129 represent the speed of light, and $t$ is time. The Lorenz inner product of $u$ and $w$ is 130 defined as:

131

$$
\langle u, w\rangle=x_{u} x_{w}+y_{u} y_{w}+z_{u} z_{w}-v^{2} t_{u} t_{w}
$$


132 Assuming there are four antennas located at $\left(x_{i}, y_{i}, z_{i}\right)$, with the associated time of arrival

133 (TOA) as $t_{i}$, where $i=1,2,3,4$ and the PD source is located at $(x, y, z)$ and has a time 134 of emission (TOE) $t$. This can presented as:

$135 s_{i}=\left[\begin{array}{c}x_{i} \\ y_{i} \\ z_{i} \\ v * t_{i}\end{array}\right], \quad s=\left[\begin{array}{c}x \\ y \\ z \\ v * t\end{array}\right]$

136 Each TOA measurement may be expressed as:

$137\left(x-x_{i}\right)^{2}+\left(y-y_{i}\right)^{2}+\left(z-z_{i}\right)^{2}=v^{2} *\left(t-t_{i}\right)^{2}$

138 Which is equivalent to:

$1392\left(x x_{i}+y y_{i}+z z_{i}-v^{2} t_{i} t\right)=x^{2}+y^{2}+z^{2}-v^{2} t^{2}+x_{i}^{2}+y_{i}^{2}+z_{i}^{2}-v^{2} t^{2}$

140 or, in vector-matrix form:

$1412 A s=\lambda 1+b$

142 Where

$143 \quad s=\left[\begin{array}{c}x \\ y \\ z \\ v^{*} t\end{array}\right]$, are the coordinates of interest

$144 \quad A=\left[\begin{array}{llll}x_{1} & y_{1} & z_{1} & -v t_{1} \\ x_{2} & y_{2} & z_{2} & -v t_{2} \\ x_{3} & y_{3} & z_{3} & -v t_{3} \\ x_{4} & y_{4} & z_{4} & -v t_{4}\end{array}\right], \lambda=\langle s, s\rangle=x^{2}+y^{2}+z^{2}-v^{2} t^{2}$ and $l=\left[\begin{array}{l}1 \\ 1 \\ 1 \\ 1\end{array}\right]$

$145 \quad b=\left[\begin{array}{c}\left\langle s_{1}, s_{1}\right\rangle \\ \left\langle s_{2}, s_{2}\right\rangle \\ \left\langle s_{3}, s_{3}\right\rangle \\ \left\langle s_{4}, s_{4}\right\rangle\end{array}\right]=\left[\begin{array}{c}x_{1}{ }^{2}+y_{1}{ }^{2}+z_{1}{ }^{2}-v^{2} t_{1}{ }^{2} \\ x_{2}{ }^{2}+y_{2}{ }^{2}+z_{2}{ }^{2}-v^{2} t_{2}{ }^{2} \\ x_{3}{ }^{2}+y_{3}{ }^{2}+z_{3}{ }^{2}-v^{2} t_{3}{ }^{2} \\ x_{4}{ }^{2}+y_{4}{ }^{2}+z_{4}{ }^{2}-v^{2} t_{4}{ }^{2}\end{array}\right]$

146 Based on equation (13), which relates $s$ to its Lorenzian norm $\lambda$, this can be rewritten as:

147 $s=\frac{1}{2} \lambda A^{-1} 1+\frac{1}{2} A^{-1} b \quad$ or $\quad s=\lambda d+e$ 


$$
d=\frac{1}{2} A^{-1} l=\left[\begin{array}{llll}
x_{d} & y_{d} & z_{d} & v t_{d}
\end{array}\right]^{T}, \quad e=\frac{1}{2} A^{-1} b=\left[\begin{array}{llll}
x_{e} & y_{e} & z_{e} & v t_{e}
\end{array}\right]^{T}
$$

150 Taking the Lorenzian norm of both sides of equation (14) results in a quadratic equation 151 in $\lambda$, i.e.

152

$$
\lambda=\langle d, d\rangle \lambda^{2}+2\langle d, e\rangle \lambda+\langle e, e\rangle \quad \text { or } \quad \alpha \lambda^{2}+\beta \lambda+\gamma=O
$$

153 Where $\gamma=\langle e, e\rangle=x_{e}{ }^{2}+y_{e}{ }^{2}+z_{e}{ }^{2}-v^{2} t_{e}^{2}$

157 Solving the quadratic equation (15) results in two solutions of $\lambda$ when $158 \alpha \neq 0$ and the possible PD solutions are located either at:

$159 \quad s_{1}=\lambda_{1} d+e=\left[\begin{array}{c}x \\ y \\ z \\ v t\end{array}\right] \quad$, or $\quad s_{2}=\lambda_{2} d+e=\left[\begin{array}{c}x \\ y \\ z \\ v t\end{array}\right]$

160 In GPS technology, the selection of a valid solution is based on clock synchronisation

161 and thus the solution with the lowest time offset (presented by $v t$ in both $s_{1}$ and $s_{2}$

162 vector) is considered to be a correct solution.

\section{Methodology}

164 The authors have developed a software platform in MATLAB that performs simulation 165 and localisation for an array of PD source positions (a grid of 64 PD positions were

166 simulated, as depicted in Figure 2). The positions were selected arbitrarily on a 167 Cartesian grid as PD sources can occur anywhere within the insulation system of HV assets. Figure 2 also shows the configuration of the antenna arrays (triangular symbols). 
170 shaped array (Figure 2b). Table 1 presents the grid coordinates of each antenna. These 171 antenna arrangement arrays were considered in a way to enable an easy setting of these 172 equipment when measurements are carried out in a real site environment, although 173 antenna arrays will generally be placed away from substation equipment to respect 174 distance clearances.
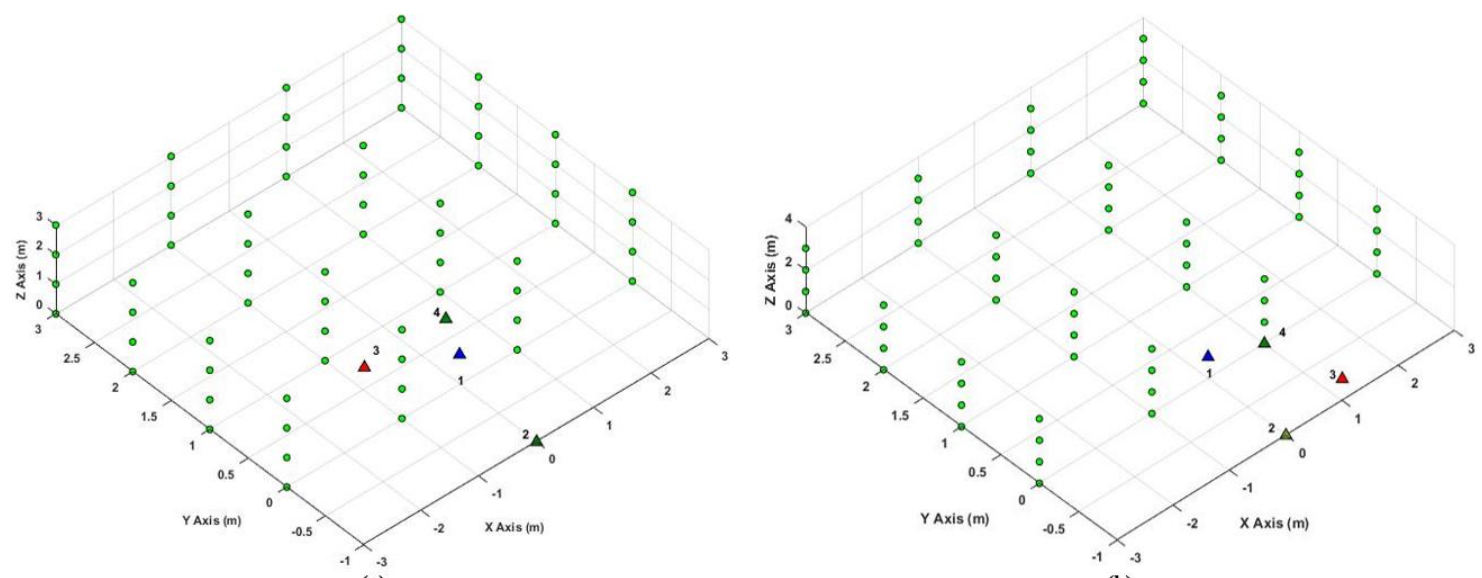

Figure 2: Simulation geometry showing PD locations (green spheres) and antenna locations (triangles) for the two array configurations (a) Y shaped array and (b) Square shaped array

Table 1: Coordinates of the antenna arrays within the simulation grid

\begin{tabular}{|c|ccc|ccc|}
\hline \multirow{2}{*}{$\begin{array}{c}\text { Antenna } \\
\text { number }\end{array}$} & \multicolumn{3}{|c|}{ Y shaped array } & \multicolumn{3}{c|}{ Square shaped array } \\
\cline { 2 - 7 } & $\boldsymbol{x}(\boldsymbol{m})$ & $\boldsymbol{y}(\boldsymbol{m})$ & $\boldsymbol{z}(\boldsymbol{m})$ & $\boldsymbol{x}(\boldsymbol{m})$ & $\boldsymbol{y}(\boldsymbol{m})$ & $\boldsymbol{z}(\boldsymbol{m})$ \\
\hline $\mathbf{1}$ & 0 & 0 & 1 & 0 & 0 & 1 \\
\hline $\mathbf{2}$ & 0 & -1 & 0 & 0 & -1 & 0 \\
\hline $\mathbf{3}$ & $-1 / \sqrt{ } 2$ & $1 / \sqrt{ } 2$ & 0 & 1 & -1 & 1 \\
\hline $\mathbf{4}$ & $1 / \sqrt{ } 2$ & $1 / \sqrt{ } 2$ & 0 & 1 & 0 & 0 \\
\hline
\end{tabular}

178 In the case of the $\mathrm{Y}$ shaped array, the respective antennae were mutually separated by a 179 distance of $1 \mathrm{~m}$, with 3 of the antennas positioned on the horizontal plane and a single 180 central antenna elevated by $1 \mathrm{~m}$ in the vertical plane. In the case of the square array, antenna positions were spaced apart by $1 \mathrm{~m}$ horizontally. Diametrically opposite antennas were offset by $1 \mathrm{~m}$ in the $z$ axis. The number of 3D PD locations was chosen based on processing time considerations. Simulated PD locations fill a defined volume 
that surrounds the antenna arrays. PD positions lie along the $x$-axis from $3 \mathrm{~m}$ to $3 \mathrm{~m}$ at intervals of $2 \mathrm{~m}$, along the $y$-axis from $0 \mathrm{~m}$ to $3 \mathrm{~m}$ at intervals of $1 \mathrm{~m}$ and along the $z$ axis from $0 \mathrm{~m}$ to $3 \mathrm{~m}$ also at intervals of $1 \mathrm{~m}$. The range of the simulated PD positions was selected so that precise appreciation of the location performance of the iterative and non-iterative algorithms was provided.

The TDOAs of the simulated PD positions were obtained using Equation 8, where $(x, y$,

$z$ ) represent the coordinates of the simulated PD position and $\left(x_{i}, y_{i}, z_{i}\right)$ the coordinates of the four antennas (1, 2, 3 and 4). The iterative algorithm (SLS) was applied and its performance evaluated, with the initial values for $(x, y, z)$ set to $(0,0,0)$. Within the iteration method, error bounds were varied from $10^{-3}$ down to $10^{-13}$ with an additional error bound defined for the time iteration and having a value of $10^{-8}$. The error bound can be defined as the incremental limit between consecutive iterations of the algorithm that produces a converged solution, thus determining the accuracy of the iterative solutions. The accuracy of the iterative method has been evaluated in terms of accuracy by comparing the difference in distance $d$ between the iterated solution to the PD location and the actual PD location. Four categories of location accuracy were defined:

- Very good accuracy: $d \leq 1 \mathrm{~cm}$

- Good accuracy: $1 \mathrm{~cm}<d \leq 50 \mathrm{~cm}$

- Poor accuracy: $50 \mathrm{~cm}<d \leq 1 \mathrm{~m}$

- Very poor accuracy: $d>1 \mathrm{~m}$

Moreover, the computational efficiencies of the algorithms were assessed by calculating the total number of iterations used to achieve converge on the stipulated error bound accuracy. This was repeated for both antenna array configurations.

207 Regarding the non-iterative methods, these are well known for providing precise estimates of the location when they are provided with accurate TDOAs [23]. In GPS, 
209 there are always uncertainties in TDOA measurements and satellite positions. These 210 inaccuracies give rise to random errors of the emitter location. However, the location 211 accuracy can be improved by solving the clock error of the receiver [24], by using 212 pseudo-range observations [22] or by limiting the TOA range based on the altitude of 213 the GPS satellites [25].

214 Determining the location of PD using non-iterative methods is a more difficult process, 215 as PD sources do not provide a time of emission to establish synchronisation with the 216 receiving sensors. In this context, results sections of the non-iterative algorithms 217 evaluate the output of the two solutions provided by these algorithms as the simulated 218 PD have accurate theoretical TOAs based on equation (1). The accuracy of the non219 iterative algorithms have been evaluated in terms of PD location by determining the difference between the calculated PD solutions (i.e. two roots solutions provided by the quadratic equations of the algorithms) and the simulated positions. Two categories were defined:

- Correct location: difference between calculated PD solution and simulated PD position equal to 0 .

- Incorrect location: difference between calculated PD solution and simulated PD position not equal to 0 .

\section{Location Performance of the Algorithms}

228 The following sections present the location results of the SLS and Bancroft algorithms using the two different antenna arrangements. The location results will be discussed in terms of location accuracy for both iterative and non-iterative methods and also the number of iterations for the SLS algorithm. 


\subsubsection{Y-shaped array}

234 To ensure converged solutions for all 64 simulated PD locations, sufficient iterations

235 were applied to the SLS algorithm for various error bounds. For the specified error 236 bounds, Figure 3 plots the number of converged PD location solutions within each of 237 the four accuracy categories defined above. It can be seen that the number of PD 238 sources located with very poor accuracy (greater than $1 \mathrm{~m}$ from the simulated locations) 239 saw a marked decrease as the error bound reduced, allowing improvement in the 240 intermediate distances and convergence towards highly accurate positions (i.e 34 241 solutions less than $1 \mathrm{~cm}$ from the true PD source position). As the error bound was 242 reduced further, no additional improvement was seen. This result demonstrates that 243 location accuracy is influenced not only by the physical arrangement of the antennas, 244 the TDOA of the signals and the accuracy of the digital sampling hardware, but also on 245 the error bound set within the location algorithm.

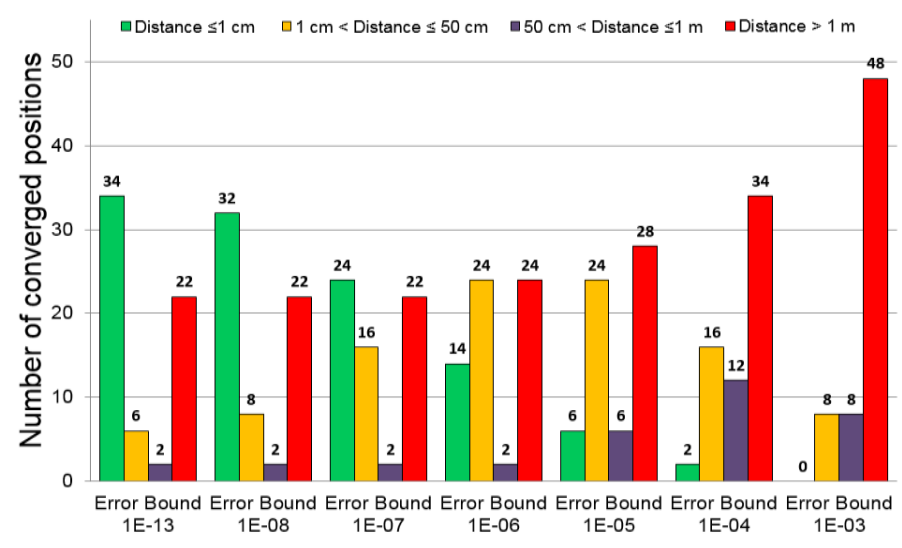

Figure 3: Number of converged PD position solutions as a function of location accuracy and error bound for simulations on the Y-shaped antenna array (SLS) 


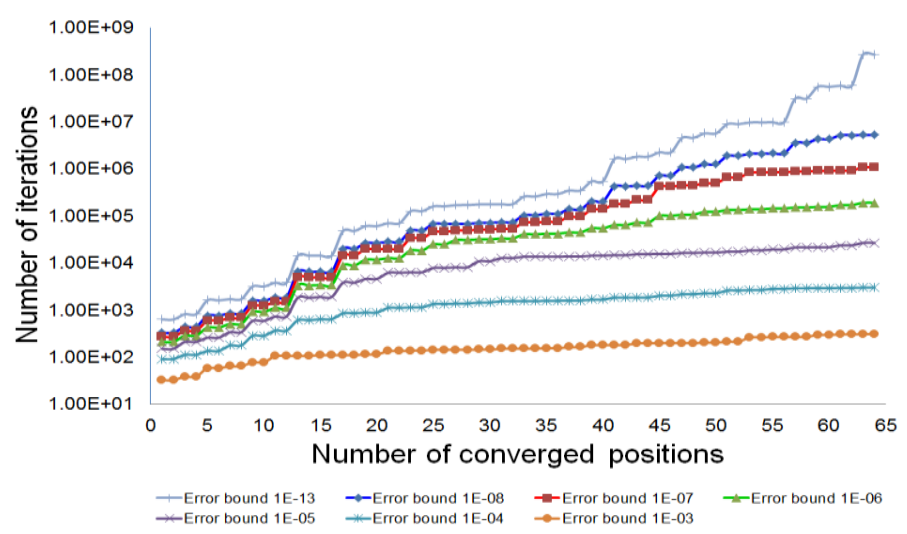

Figure 4: Results of simulations on the Y-shaped antenna array showing number of iterations vs. number of converged PD positions for various error bounds (SLS).

246 Figure 4 plots the total number of iterations needed for solutions to converge on all 64

247 PD locations for the seven error bounds under consideration. This result demonstrates

248 the relationship between the number of iterations and the error bound, with the former 249 increasing significantly from a few hundred to hundreds of millions as the error bound 250 decreases. Such a large number of iterations has the consequence of increasing 251 computational time from a few seconds to several hours using a standard desktop 252 machine (computation of these results were carried out using an Intel Q6600 Core2 253 Quad 2.4 GHz Processor). Extended computing times would be impractical if location 254 were required in real-time or close to real-time.

255 The percentage of PD sources pinpointed within the defined accuracy limits is shown in 256 Table 2 together with the number of iterations performed for each respective error 257 bound. It is clear from Table 2 that the location accuracy improves as the error bound 258 decreases. Consequently, the iterative steps accumulate in number. Additionally, using 259 the lowest error bound i.e. $10^{-13}$, which was found to be the best possible accuracy for 260 this arrangement, the number of PDs located at more than $1 \mathrm{~m}$ from the simulated 261 positions was found to be slightly high. This is due to the spatial separation between the 262 different antennas and the antenna arrangement as further results using the square 263 antenna arrangement shows improved location accuracy. 
Table 2: Results of SLS algorithm showing percentage of solutions converging within the defined location accuracy limits for the Y-shaped antenna array.

\begin{tabular}{cccccc}
\hline Error Bound & $\mathbf{d} \leq \mathbf{1} \mathbf{~ c m}$ & $\mathbf{1} \mathbf{c m}<\mathbf{d} \leq \mathbf{5 0} \mathbf{~ c m}$ & $\mathbf{5 0} \mathbf{c m}<\mathbf{d} \leq \mathbf{1} \mathbf{~ m}$ & $\mathbf{d}>\mathbf{1} \mathbf{~ m}$ & No. of Iterations \\
\hline $\mathbf{1 0}^{-\mathbf{1 3}}$ & $53.1 \%$ & $9.4 \%$ & $3.1 \%$ & $34.4 \%$ & 275740268 \\
\hline $\mathbf{1 0}^{-\mathbf{0 8}}$ & $50.0 \%$ & $12.5 \%$ & $3.1 \%$ & $34.4 \%$ & 5371396 \\
\hline $\mathbf{1 0}^{-\mathbf{0 7}}$ & $37.5 \%$ & $25 \%$ & $3.1 \%$ & $34.4 \%$ & 1104646 \\
\hline $\mathbf{1 0}^{-\mathbf{0 6}}$ & $21.9 \%$ & $37.5 \%$ & $3.1 \%$ & $37.5 \%$ & 194065 \\
\hline $\mathbf{1 0}^{-\mathbf{- 0 5}}$ & $9.4 \%$ & $37.5 \%$ & $9.4 \%$ & $43.8 \%$ & 27325 \\
\hline $\mathbf{1 0}^{-\mathbf{0 4}}$ & $3.1 \%$ & $25 \%$ & $18.8 \%$ & $53.1 \%$ & 3164 \\
\hline $\mathbf{1 0}^{-\mathbf{0 3}}$ & $0.0 \%$ & $12.5 \%$ & $12.5 \%$ & $75.0 \%$ & 315 \\
\hline
\end{tabular}

Table 3: Results of SLS algorithm showing percentage of solutions converging within the defined location accuracy limits for the square-shaped antenna array.

\begin{tabular}{cccccc}
\hline Error Bound & $\mathbf{d} \leq \mathbf{1} \mathbf{~ c m}$ & $\mathbf{1} \mathbf{c m}<\mathbf{d} \leq \mathbf{5 0} \mathbf{~ c m}$ & $\mathbf{5 0} \mathbf{c m}<\mathbf{d} \leq \mathbf{1} \mathbf{~ m}$ & $\mathbf{d}>\mathbf{1} \mathbf{~ m}$ & No. of Iterations \\
\hline $\mathbf{1 0}^{-13}$ & $95.3 \%$ & $0 \%$ & $0 \%$ & $4.7 \%$ & 2212354990 \\
\hline $\mathbf{1 0}^{-08}$ & $84.4 \%$ & $10.9 \%$ & $0 \%$ & $4.7 \%$ & 11755016 \\
\hline $\mathbf{1 0}^{-07}$ & $67.2 \%$ & $26.6 \%$ & $1.6 \%$ & $4.7 \%$ & 1727533 \\
\hline $\mathbf{1 0}^{-06}$ & $29.7 \%$ & $57.8 \%$ & $6.3 \%$ & $6.3 \%$ & 243296 \\
\hline $\mathbf{1 0}^{-05}$ & $7.8 \%$ & $60.9 \%$ & $15.6 \%$ & $15.6 \%$ & 55905 \\
\hline $\mathbf{1 0}^{-04}$ & $1.6 \%$ & $34.4 \%$ & $15.6 \%$ & $48.4 \%$ & 4140 \\
\hline $\mathbf{1 0}^{-03}$ & $0 \%$ & $18.8 \%$ & $15.6 \%$ & $65.5 \%$ & 263 \\
\hline
\end{tabular}

\subsubsection{Square-shaped array}

265 The results obtained using SLS with the square antenna array proved similar to those obtained previously with regards to the accuracy and number of iterations (See Figure 5 and Figure 6). With an error bound of $10^{-03}, 42 \mathrm{PD}$ positions were located with very poor accuracy (metres from their true position). The number of PD located $>1 \mathrm{~m}$ from the simulated positions was reduced significantly as the error bound became smaller,

270 allowing the intermediate distances to improve and solutions to converge towards very 271 accurate locations of less than $1 \mathrm{~cm}$ from the true PD source position. However, Table 3

272 shows a considerable improvement of the location accuracy. At an error bound of $10^{-13}$, $27395.3 \%$ of iterated PD positions were to within an accuracy of less than $1 \mathrm{~cm}$. Whereas, 274 in the case of the Y-shaped array configuration, only 53.1\% of PD were located to 275 within the same accuracy at the same error bound. The 3 remaining PD positions 
276 located at a distance of $>1 \mathrm{~m}$ did not show any further improvement despite further 277 reduction in the error bound. The non-location of these PD positions was mainly due to 278 the applied initial value $(0,0,0)$ since, after replacing those initial values by the actual 279 true value of the PD locations, calculation provided a correct solution.

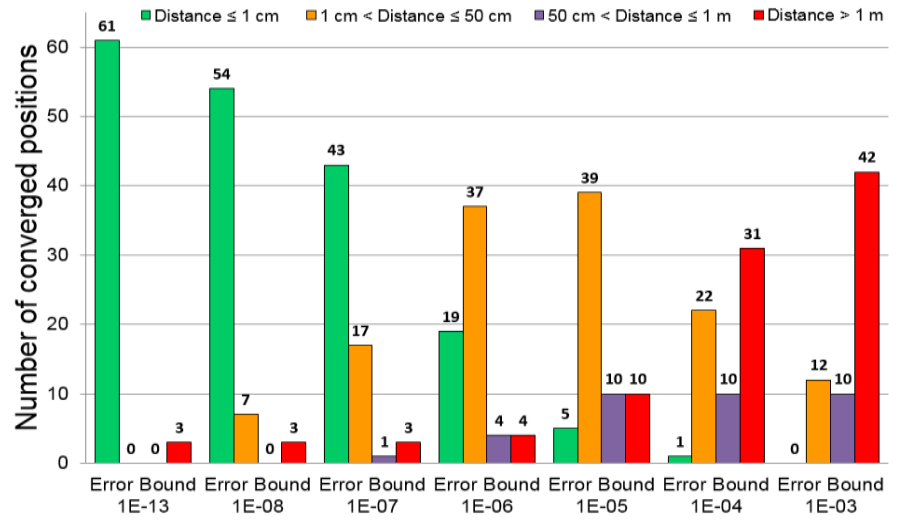

Figure 5: Number of converged positions as a function of both location accuracy and error bound for square shaped arrangement (SLS)

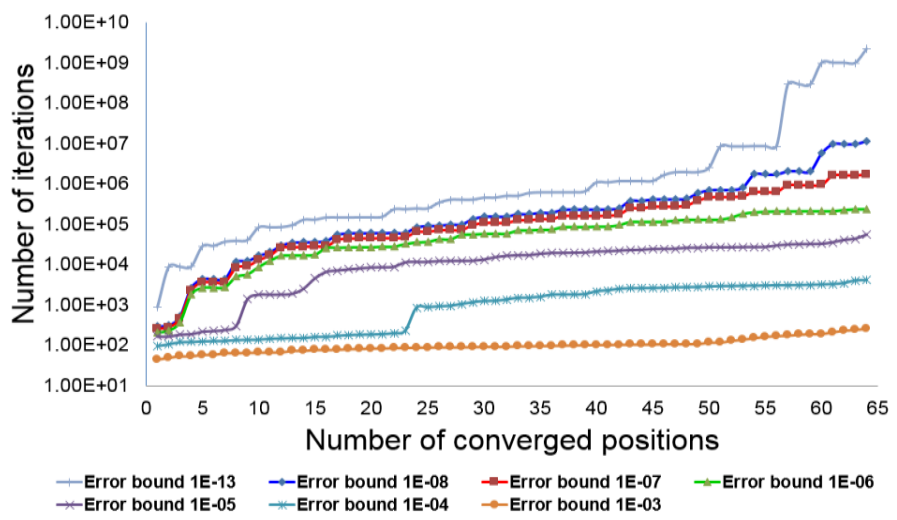

Figure 6: Results of simulations on the square-shaped antenna array showing number of iterations vs. number of converged PD positions for various error bounds (SLS).

\subsubsection{Discussion}

281 As shown in Table 2 and Table 3, which present respectively the effectiveness of the Y and square shape arrays to locate PD occurring at each of the 64 grid positions, it can be seen that in the case of the square array, $95.3 \%$ of the converged solutions locate PD to within $1 \mathrm{~cm}$ of their true position at an error bound of $10^{-13}$. In contrast, the Y shaped 

position at the same error bound, which represents the best possible accuracy for this arrangement in the present study. These results show that in addition to the influence of the algorithms' error bound and the number of iteration on the location accuracy, 289 antenna arrangement are also key for enhanced location results. This is mostly due to 290 the square antenna arrangement having a better spatial separation and better coverage area than the Y shaped antenna arrangement.

292 In Figure 7 which shows the number of PD positions located with an accuracy of $1 \mathrm{~cm}$ 293 or less as a function of error bound, one may conclude that, while requiring more 294 iterations, the SLS algorithm as applied to PD location using the square array, generally 295 produces more accurate results than with the Y shaped array (see Figure 8).

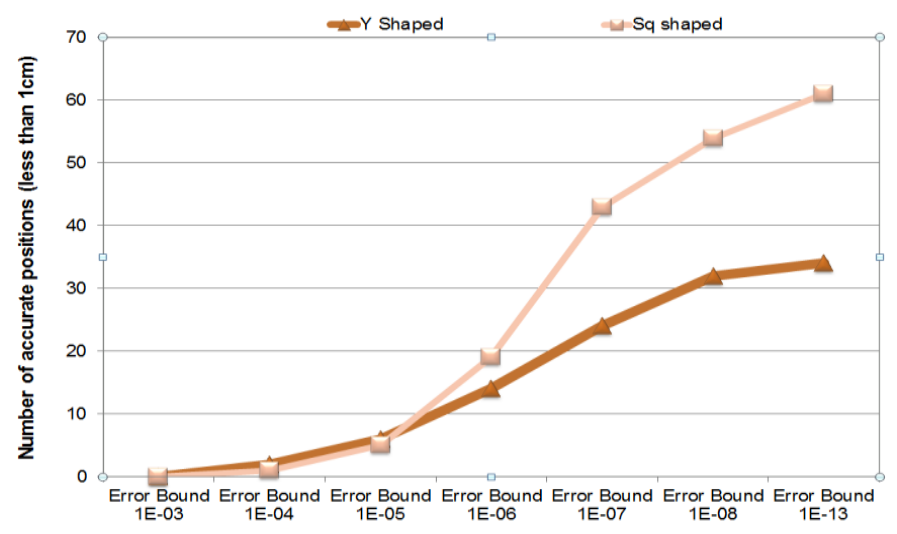

Figure 7: Number of accurate PD location solutions $(<1 \mathrm{~cm}$ from the PD source) for the two array configurations as a function of error bound (SLS)

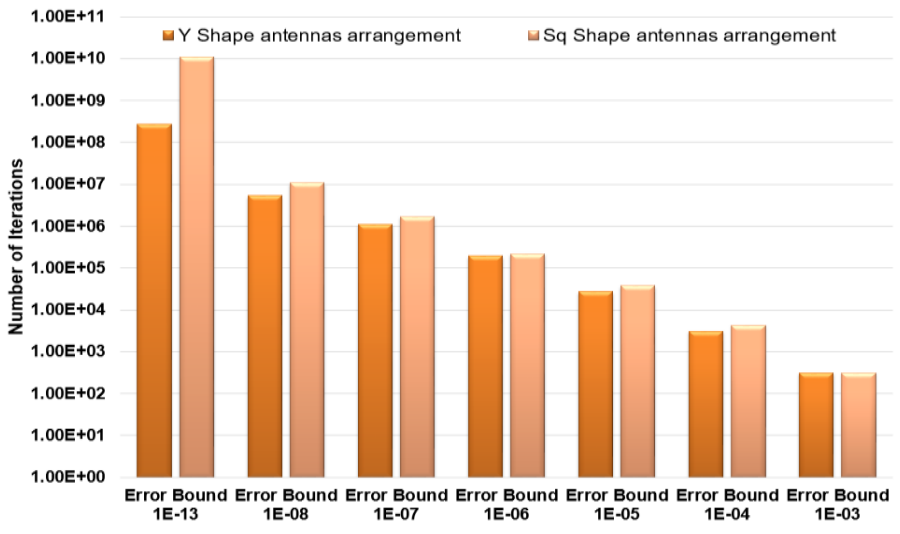


Figure 8: Number of iterations required to achieve converged solutions for each antenna configuration as a function of error bound (SLS)

296

297

298

299

300

301

302

303

304

305

306

307

308

309

\subsection{Bancroft algorithm}

Bancroft [21] determined a closed form expression for global positioning system pseudo-range equations. In his derivation of the formula, Bancroft made use of the Lorentz inner product and demonstrated that pseudo-range equations are hyperbolic in nature and may have two solutions. Although he did explicitly discuss the GPS navigation solution which determines the coordinates $(x, y, z)$ and the clock offset of a GPS receiver, the understanding of the two solutions provided by the algorithm with regard to partial discharge location using RF technique is investigated in the following paragraphs.

\subsubsection{Y shaped antenna array}

To evaluate the performance of the two solutions provided by the Bancroft algorithm, the $64 \mathrm{PD}$ positions defined on the simulation grid were computed by the Bancroft algorithm as described in Section 3. Figure 9 presents the number of correct and incorrect location solutions provided by both the positive and negative root.

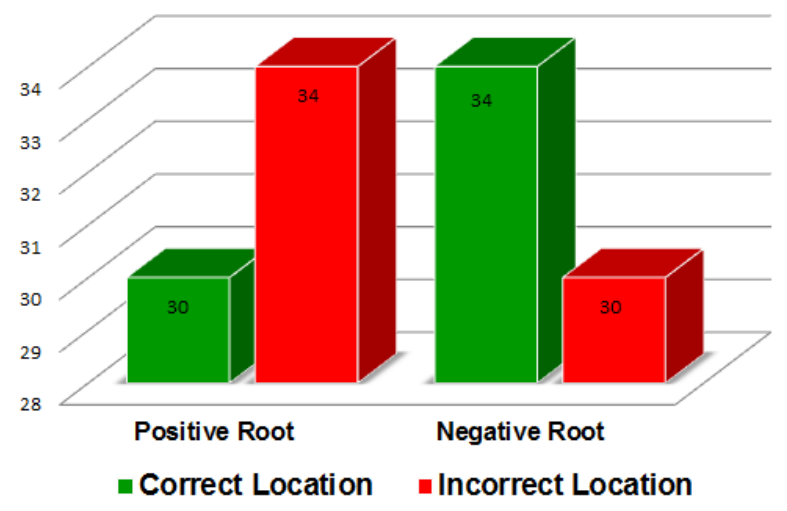

Figure 9: Location results of Bancroft algorithm using Y shaped antenna arrangement 


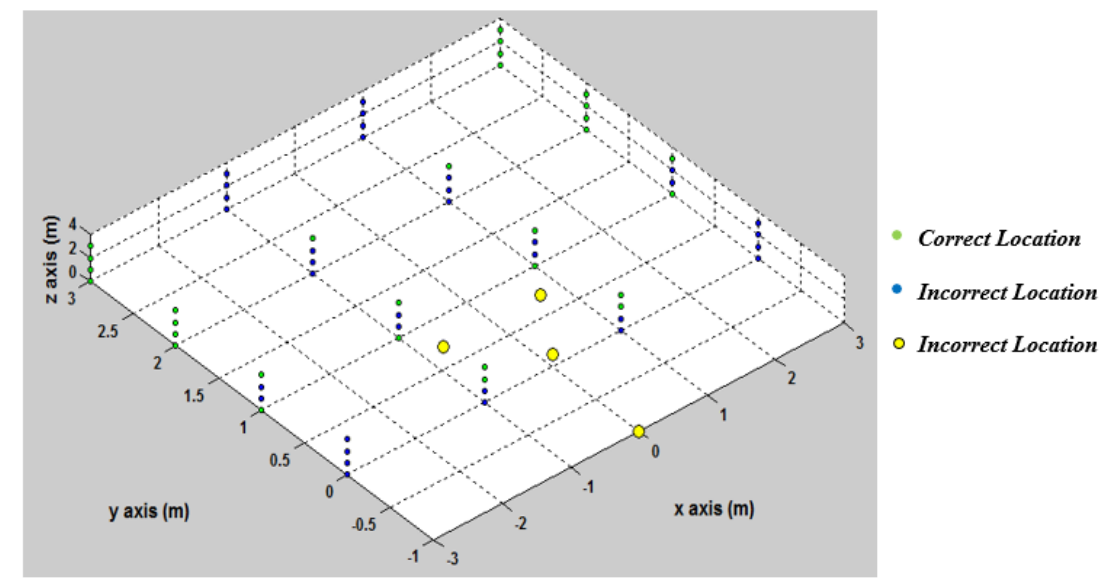

Figure 10: Position of located and non-located PD using Y shaped antenna arrangement and positive root of the Bancroft algorithm

310 Based on results of the positive root of the Bancroft algorithm, it can be seen that the 311 algorithm provided accurate positioning to 30 PD locations and 34 incorrect solutions to 312 the remaining PD positions. This demonstrates that the algorithm can only provide 313 partial results to the 64 simulated PD using one of the roots and that the location of 314 these simulated PD require the investigation of both solutions.

315 The exact position of the located and non-located PD is presented in Figure 10, where 316 the green points represent the located positions and the blue points the incorrect 317 solutions. It can be seen from the figure that the positioning results of located and non318 located PD positions are symmetrical around the antenna central point. This is due to 319 the topology of the Y shaped array, of which the $y$ and $z$ coordinates of antennas 3 and 4 320 are identical.

321 Regarding the location results of the Bancroft algorithm using the negative root, it can 322 be seen from Figure 9 that the algorithm provided 34 accurate PD locations and 30 323 inaccurate PD locations. It should be noted that inaccurate locations using the positive 324 root are found to be located accurately using the negative root and vice versa. This 325 demonstrates that the algorithm can provide accurate locations to the 64 simulated PD 
326 positions if valid solutions are selected between both roots. This demonstrates that the 2

327 solutions provided by the algorithm complement each other to provide accurate

328 positioning to the simulated PD. This is because the two hyperbolas intersect at two

329 locations, one that corresponds to the TDOA with correct sign and the other to the

330 TDOA with reversed sign.

331

332

333

\subsubsection{Square shaped antenna arrangement}

Using the square antenna arrangement and the positive root, the Bancroft algorithm provided 17 correct locations and 47 incorrect locations (see Figure 11). On the other hand, positioning results using the negative root provided more accurate locations than the positive root, where 52 out of the 64 simulated PD positions located accurately and only 12 PD were located incorrectly. The difference between the correct PD locations using the positive root and the non-located PD using the negative root results from 5 PD positions being located accurately by both roots.

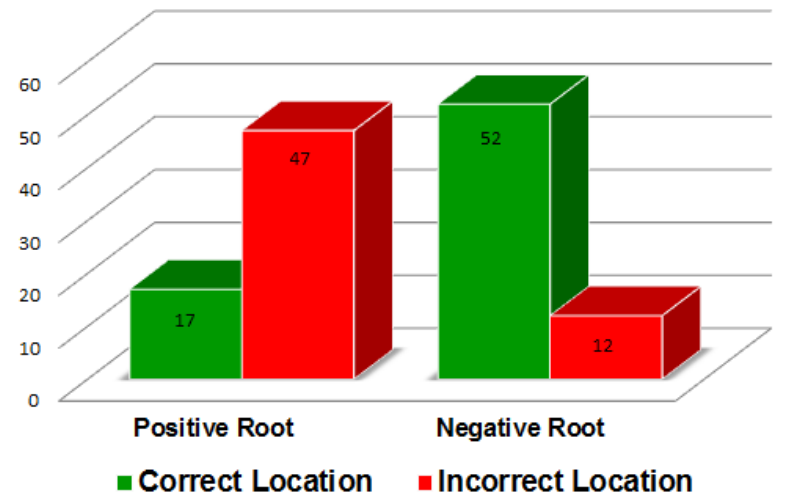

Figure 11: Location results of Bancroft algorithm using square shaped antenna arrangement

339

340

341

\subsubsection{Discussion}

Based on the results of the Bancroft algorithm using both positive and negative roots, it can be seen that the algorithm can provide very accurate location results on the 64 simulated PD positions. Results also show that the algorithm provided more successful location results when using the negative root instead of the positive root. In addition, 
344 location results using the square antenna arrangement were found to be better than the

345 location results when using the $\mathrm{Y}$ shaped antenna arrangement. Although location 346 results using the different antenna arrangements differ in terms of the number of 347 successfully located PD using each root, the discrimination between correct and 348 incorrect solutions of the positive and negative root can be carried out using the clock 349 offset parameter. Based on the simulated PD, it was found that the Bancroft algorithm can provide $100 \%$ accurate solutions to the simulated PD positions when selecting the cartesian coordinates $(x, y, z)$ corresponding to the lowest clock offset when comparing results of both roots. Validation of this selection process may change when considering noise effects and measurement errors as time offset adjustments cannot be established 354 due to the stochastic nature of the physical PD emission process.

355 Additionally, given only the difference in arrival times of the antennas' signals, it is difficult to know which solution is correct. The separation between the algorithm's correct and incorrect solutions will depend on the environment where measurements took place. For example, in the case where measurements are carried out in a high voltage power transformer using acoustic sensors attached to the transformer's housing, discrimination between the different solutions can use the equipment's area spatial volume to limit the search of valid solutions. In the case of open space areas such as electrical substations, if the reference point is at the ground height and the locations of interest are in front of the antenna arrangements, one can limit the search of valid solutions within the positive interval of $\mathrm{y}$ and $\mathrm{z}$ coordinates.

\section{Comparison between Iterative and Non-Iterative Algorithms}

366 Nonlinear equations of location algorithms which are presented by hyperbolas and 367 distance formulas are commonly solved with iterative algorithms [26]. Results of the iterative algorithm showed that these methods have strong dependencies on different 
parameters such as the error bound, number of iterations and also initial values which must be provided by the user. On the other hand, non-iterative methods, which do not require iterations and therefore make a fast computation tool, showed that they provide very accurate location results when provided by accurate TDOAs (in this case,

373 theoretical TDOAs were provided). However the selection of correct locations among 374 the two available solutions will depend on the user's experience and ability to 375 discriminate between the different positioning solutions by using for example time restrictions based on the equipment's spatial volume. Table 4 presents some of the advantages and disadvantages of the different location algorithms when applied to PD location.

Table 4: Characteristics of the location algorithms

\begin{tabular}{|c|c|c|}
\hline Algorithm & Advantages & Disadvantages \\
\hline 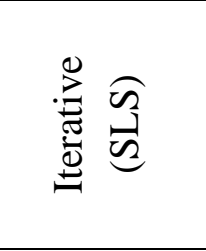 & $\begin{array}{ll}\text { - } & \text { Accurate if provided with well } \\
\text { selected error bound } \\
\text { - } & \text { Accurate if provided with well } \\
\text { selected number of iterations } \\
\text { - Accurate if provided with accurate } \\
\text { time of arrival }\end{array}$ & $\begin{array}{l}\text { - } \\
\text { - Depends on number of iterations } \\
\text { - Depends on error bound } \\
\text { - Depends on initial values } \\
\end{array}$ \\
\hline 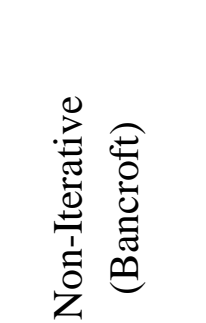 & $\begin{array}{l}\text { - } \text { Direct solution } \\
\text { - } \text { Fast and very accurate } \\
\text { - Do not depend on initial values } \\
\text { - Possibility of discriminating between } \\
\text { the two solutions (Bancroft method } \\
\text { only) }\end{array}$ & $\begin{array}{l}\text { - } \\
\text { - }\end{array}$ \\
\hline
\end{tabular}

379 Using iterative methods, the question which is still raised is: how can the user define a 380 valid error bound and also a valid number of iterations sufficient to provide accurate 381 location results assuming there is no initial values issue (see example of SLS performance at $10^{-13}$ error bound in Figure 5)? 


\section{New Approach}

384 Based on simulations, it was found that when the error bound is high (e.g. $10^{-3}$ error 385 bound), solutions of the location coordinates are often underestimated and the number 386 of iterations required is also low. When the location coordinates of some TDOAs using 387 the iterative results are compared to the location coordinates of the same TDOAs using 388 non-iterative methods, this may show a location mismatch in the case of a non-valid 389 error bound selection and which indicates that the error bound should be decreased. 390 This process should be repeated until matching results are found by both iterative and 391 non-iterative methods. Regarding the selection of a valid number of iterations, this is 392 determined by providing enough iteration values which allow a converged solution 393 based on the matching solutions of both iterative and non-iterative methods to be 394 obtained. Figure 12 summarises the selection process of valid error bounds and number 395 of iterations used by the iterative methods based on the non-iterative method solutions. 396 It should be noted that the iterative methods may sometimes provide a non-converged 397 solutions which may be due to initial values issue or measurement errors. 


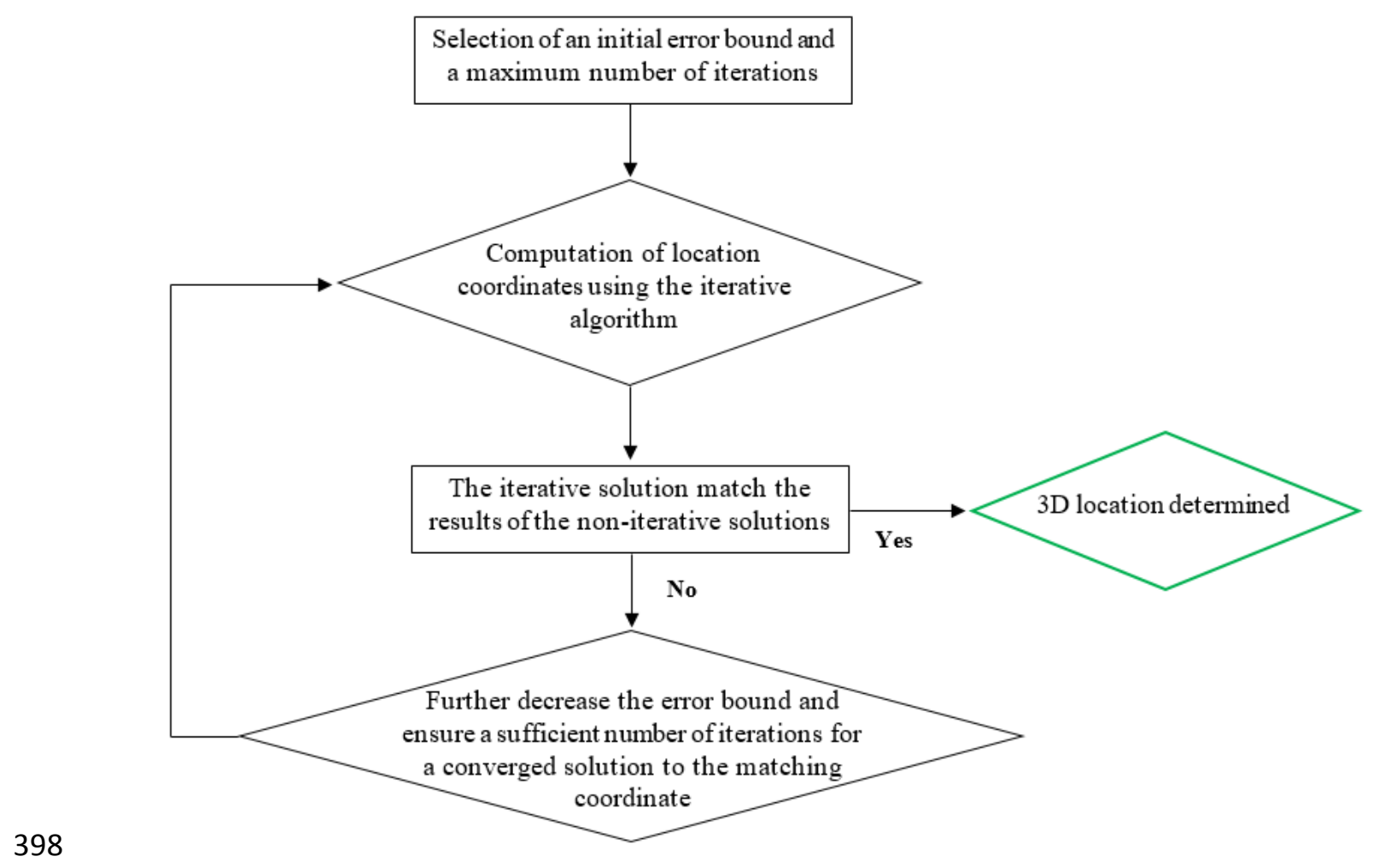

Figure 12: Selection of error bound and number of iterations

\section{Conclusions}

401 As a study evaluating the location accuracy of an iterative and non-iterative algorithms

402 as applied to partial discharge measurement, simulations of a range of PD using two

403 different antenna configurations have been presented.

404 By varying the error bounds, it has been shown that the performance of the iterative 405 algorithms as a function of location accuracy can be quantified, despite the nonlinear 406 nature of the location equations. A decrease in the error bound produces more accurate 407 location results while requiring more iterations. The results presented will be useful for 408 a practitioner of condition monitoring of in-service power equipment since it will allow 409 judgement of appropriate levels of required accuracy based on the dimensions of the 410 equipment under surveillance. It will also facilitate estimation of the required 411 computing time to achieve the desired level of location accuracy. The required spatial 412 location accuracy depends on the application. For example, general surveying of 
413 equipment on a substation-wide scale may only require a poor to good level of accuracy

$414(1 \mathrm{~cm} \leq \mathrm{d} \leq 1 \mathrm{~m})$. This range may also accurately facilitate the location of faults along 415 large equipment sections such as busbars, bushings or power transformers (i.e. larger 416 equipment).

417 Regarding the non-iterative algorithms, it was found that these techniques provide very 418 accurate positioning when provided with precise TDOAs. The accuracy of the non419 iterative algorithms also depends on the antenna arrangements which influence the 420 number of accurate positions located by the two different roots. The discrimination 421 process between the two different solutions of the non-iterative solutions can be 422 difficult and will depend on the user experience to separate between the two solutions 423 using, for example, time restrictions based on the equipment's spatial volume.

424 A novel approach to select adequate error bounds and number of iterations using results 425 of the non-iterative methods has been established and will contribute considerably to 426 solve some of the iterative method dependencies.

427 In this work, simulations provided an evaluation of the performance of different types 428 of location algorithms based on determined PD locations. This evaluation method gives 429 indications of the essential characteristics of iterative methods and also an insight on the 430 behaviour of non-iterative methods to provide different solutions. The study presented 431 in this paper can benefit electrical utilities, network operators and designers of PD 432 locations systems, as it can be used as a guide to the selection of specific algorithm 433 based on its operation requirements (i.e. computation time, discrimination between 434 solutions, accuracy parameters and their selection process), facilitating more accurate 435 location and diagnosis of incipient faults in high value electrical power equipment. 


\section{Acknowledgements}

437 The work presented in this paper were obtained as part of a financial, academic and 438 technical support provided by Glasgow Caledonian University during the main author $439 \quad \mathrm{PhD}$ studies. 


\section{References}

[1] Moore, P. J., Portugues, I. E., and Glover, I. A. "Radiometric location of partial discharge sources on energized high-voltage plant" IEEE Transactions on Power Delivery, 2005, 20, (3), pp 2264-2272

[2] Stewart, B. G., Nesbitt, A., and Hall, L. "Triangulation and 3D location estimation of RFI and partial discharge sources within a $400 \mathrm{kV}$ substation" Proceedings on IEEE Electrical Insulation Conference, Montreal, Canada, June 2009, pp 164-168

[3] Tian, Y., Kawada, M., and Isaka, K. "Locating partial discharge source occurring on distribution line by using FDTD and TDOA methods" IEEJ Transactions on Fundamentals and Materials, 2009, 129, (2), pp 89-96

[4] El Mountassir, O. Stewart, B, G. McMeekin, S. G. and Ahmadinia, A. "Evaluation of an iterative method used for partial discharge RF location techniques", $10^{\text {th }}$ International Conference on Environment and Electrical Engineering, 2011, pp 1-4

[5] Coenen, S. and Tenbohlen, S. "Location of PD sources in power transformers by UHF and acoustic measurements" IEEE Transactions on Dielectrics and Electrical Insulation, 2012, 19, pp 1934-1940

[6] Steiner, J. P., Reynolds, P. H., and Weeks, W. L. "Estimating the location of partial discharges in cables” IEEE Transactions on Electrical Insulation, 1992, 27, pp 44-59

[7] Tian, Y., Lewin, P., Davies, A., Sutton, S., and Swingler, S. "Partial discharge detection in cables using VHF capacitive couplers" IEEE Transactions on Dielectrics and Electrical Insulation, 2003, (10), pp 343-353 
464 [8] Mardiana, R. and Su, C. Q. "Partial discharge location in power cables using a phase difference method" IEEE Transactions on Dielectrics and Electrical Insulation, 2010, (17), pp 1738-1746

[9] Wagenaars, P., Wouters, P. A. A. F., Van der Wielen, P. C. J. M., and Steennis,

[12] Hikita, M., Ohtsuka, S., Wada, J., Okabe, S., Hoshino, T., and Maruyama, S. "Study of partial discharge radiated electromagnetic wave propagation characteristics in an actual $154 \mathrm{kV}$ model GIS" IEEE Transactions on Dielectrics and Electrical Insulation, 2012, (19), pp 8-17

[13] Chiu, M. Y., Liang, K.W., Lee, C. H., Fan, C. L., Cheng, J., and C., L. Y. "Application of MV GIS partial discharge measurement and location" International Conference on Condition Monitoring and Diagnosis, 2012, pp 462465

[14] Zheng, B and Bojovschi, A. "Electromagnetic Sensing of Partial Discharge in Air-insulated Medium Voltage Switchgear", IEEE Power Engineering and Automation Conference, 2012, pp 1-4 
[15] Judd, M. D., Yang, L., and Hunter, I. B. B. "Partial discharge monitoring for power transformer using UHF sensors. Part 2: Field experience" IEEE Electrical Insulation Magazine, 2005, (21), pp 5-13

[16] Tang, Z. G., Chengrong, L. R., Xu, C. ; Wei, W., Jinzhong, L., and Jun, L. "Partial discharge location in power transformers using wideband RF detection" IEEE Transactions on Dielectrics and Electrical Insulation, 2006, (13), pp 11931199

[17] Sinaga, H. H., Phung, B. T., and Blackburn, T. R. "Partial discharge localization in transformers using UHF detection method" IEEE Transactions on Dielectrics and Electrical Insulation, 2012, (19), pp 1891-1900

[18] Zhang, X., Tang, J., and Xie, Y. "Taylor-genetic algorithm on PD location" International Conference on High Voltage Engineering and Application, 2008, pp 685-688

[19] Miao, P., Li, X., Huijuan, H., Gehao, S., Hu, Y., and Jiang, X. "Location algorithm for partial discharge based on radio frequency (RF) antenna array" Asia-Pacific Power and Energy Engineering Conference, 2012, pp 1-4

[20] Y. Lu, X. Tan, X. Hu, "PD detection and localisation by acoustic measurements in an oil-filled transformer", IEEE Transactions on Science, Measurement and Technology, 2002, (147), pp 81-85

[21] O. El Mountassir, B. G. Stewart, S. G. McMeekin and A. Ahmadinia. "Effect of sampling rate on the location accuracy of measurements from radiated RF partial discharges signals" $11^{\text {th }}$ International Conference on Environment and Electrical Engineering, 2011, pp 891-896

[22] Bancroft, S. "An algebraic solution of the GPS equations" IEEE Transactions on Aerospace and Electronic Systems, 1985, (21), pp 56-59 
513 [23] Ho, K. M. and Chan, Y. T. "Solution and performance analysis of geolocation by TDOA" IEEE Transactions on Aerospace and Electronic Systems, 1993, (29), pp 1311-1322

516 [24] Yang, M. and Chen, K. H. "Performance assessment of a noniterative algorithm 517 for global positioning system (GPS) absolute positioning" Procceedings of the 518 National Science Council, 2001, (25), pp 102-106

519 [25] Bucher, R. and Misra, D. "A synthesizable VHDL model of the exact solution 520 for three-dimensional hyperbolic positioning system” VLSI Design Journal, 2002, (15), pp 507-520

522 [26] Markalous, S., Tenbohlen, S., and Feser, K. "Detection and location of partial 523 discharges in power transformers using acoustic and electromagnetic signals" IEEE Transactions on Dielectrics and Electrical Insulation, 2008, (15), pp 1576- 


\section{Research Highlights}

- The location performance of an iterative and non-iterative algorithms is proposed

- Iterative methods depend on error bound, number of iterations and antenna array

- Decrease of the error bound results in increased location accuracy and iterations

- Non-iterative methods provide accurate location results if the TDOAs are accurate 


\section{Response to Reviewers}

\begin{tabular}{|c|c|c|}
\hline & \multicolumn{2}{|c|}{$\begin{array}{l}\text { Quantification of the Performance of Iterative and Non-Iterative Computational } \\
\text { Methods of Locating Partial Discharges Using RF Measurement Techniques }\end{array}$} \\
\hline \multicolumn{3}{|c|}{ Reviewer \#1 } \\
\hline & Comments & Responses \\
\hline & \multicolumn{2}{|l|}{ No additional comments } \\
\hline \multicolumn{3}{|c|}{ Reviewer \#2 } \\
\hline & \multicolumn{2}{|c|}{ The reviewer has accepted the paper in the last revision. Non additional changes are requested } \\
\hline \multicolumn{3}{|c|}{ Reviewer \#3 } \\
\hline \multicolumn{3}{|c|}{ Editorial comments } \\
\hline 1 & Line 90: do not write the unit in italic & \multirow{7}{*}{$\begin{array}{l}\text { All edits and changes to figures were addressed as } \\
\text { per by the reviewer recommendations. }\end{array}$} \\
\hline 2 & $\begin{array}{l}\text { Line 95: indices should not be in italic, } \\
\text { except they are variables }\end{array}$ & \\
\hline 3 & $\begin{array}{l}\text { Line 96: } \mathrm{n} \text { is a variable and should be } \\
\text { written in italic } \mathrm{n}\end{array}$ & \\
\hline 4 & $\begin{array}{l}\text { Figure 2: quality of Fig. } 2 \text { is not good and } \\
\text { could be improved }\end{array}$ & \\
\hline 5 & Table 3: place the table on one side & \\
\hline 6 & $\begin{array}{l}\text { Fig. 8: legend is difficult to read; use } \\
\text { different colors for the type of antennas }\end{array}$ & \\
\hline 7 & $\begin{array}{l}\text { Fig. 12: quality of Fig. } 12 \text { is not good and } \\
\text { could be improved }\end{array}$ & \\
\hline \multicolumn{3}{|c|}{ Technical Comments } \\
\hline 1 & Equ.13: What is $s$ in this equation? & $\begin{array}{l}\text { s is the location of interest and is defined in equation } \\
10 . \text { To avoid confusion, the definition of all the } \\
\text { parameters of equation } 13 \text { now includes variable "s". }\end{array}$ \\
\hline
\end{tabular}


Offshore Renewable Energy Catapult

121 George Street

Glasgow

G1 1RD

UK

$11^{\text {th }}$ October 2016

Dear Editor,

Please find attached a revised copy of the manuscript entitled "Quantification of the Performance of Iterative and Non-Iterative Computational Methods of Locating Partial Discharges Using RF Measurement Techniques". This manuscript is submitted for consideration for publication as an original research paper within the Electric Power Systems Research.

We hereby confirm that this manuscript is our original work, has not been published previously and it is not being reviewed for publication by another organisation.

Yours faithfully,

Dr Othmane El Mountassir

Project Engineer

Telephone: (+44) 3330041346

E-mail: othmanee.el.mountassir@ore.catapult.org.uk 